\title{
(息)
}

Citation:

Gledhill, A and Murray, E and Forsdyke, D (2017) Psychological interventions associated with injury prevention: A systematic review. In: IOC Conference on the Prevention of Injury and Illness in Sport, 16 March 2017 - 18 March 2017, Monaco.

Link to Leeds Beckett Repository record:

https://eprints.leedsbeckett.ac.uk/id/eprint/3597/

Document Version:

Conference or Workshop Item (Presentation)

The aim of the Leeds Beckett Repository is to provide open access to our research, as required by funder policies and permitted by publishers and copyright law.

The Leeds Beckett repository holds a wide range of publications, each of which has been checked for copyright and the relevant embargo period has been applied by the Research Services team.

We operate on a standard take-down policy. If you are the author or publisher of an output and you would like it removed from the repository, please contact us and we will investigate on a case-by-case basis.

Each thesis in the repository has been cleared where necessary by the author for third party copyright. If you would like a thesis to be removed from the repository or believe there is an issue with copyright, please contact us on openaccess@leedsbeckett.ac.uk and we will investigate on a case-by-case basis. 


\section{Psychological Interventions Associated with Injury Prevention: A Systematic Review}

Dr Adam Gledhill: Carnegie School of Sport, Leeds Beckett University, Leeds, UK. Twitter: @gleds13

Eliot Murray: School of Clinical and Applied Sciences, Leeds Beckett University, Leeds, UK. Twitter: @eliot_murray

Dale Forsdyke: School of Sport, York St. John University, York, UK Twitter: @forsdyke_dale 


\section{REVIEW}

\section{Introduction}

Injuries are multifactorial. Our intervention programmes should be the same.

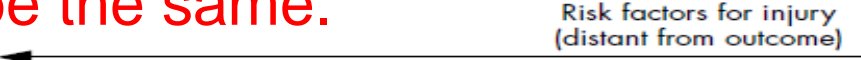

Understanding injury mechanisms: a key component of preventing injuries in sport

\section{R Bahr, T Krosshaug}

Br J Sports Med 2005;39:324-329. doi: 10.1 136/bjsm.2005.018341

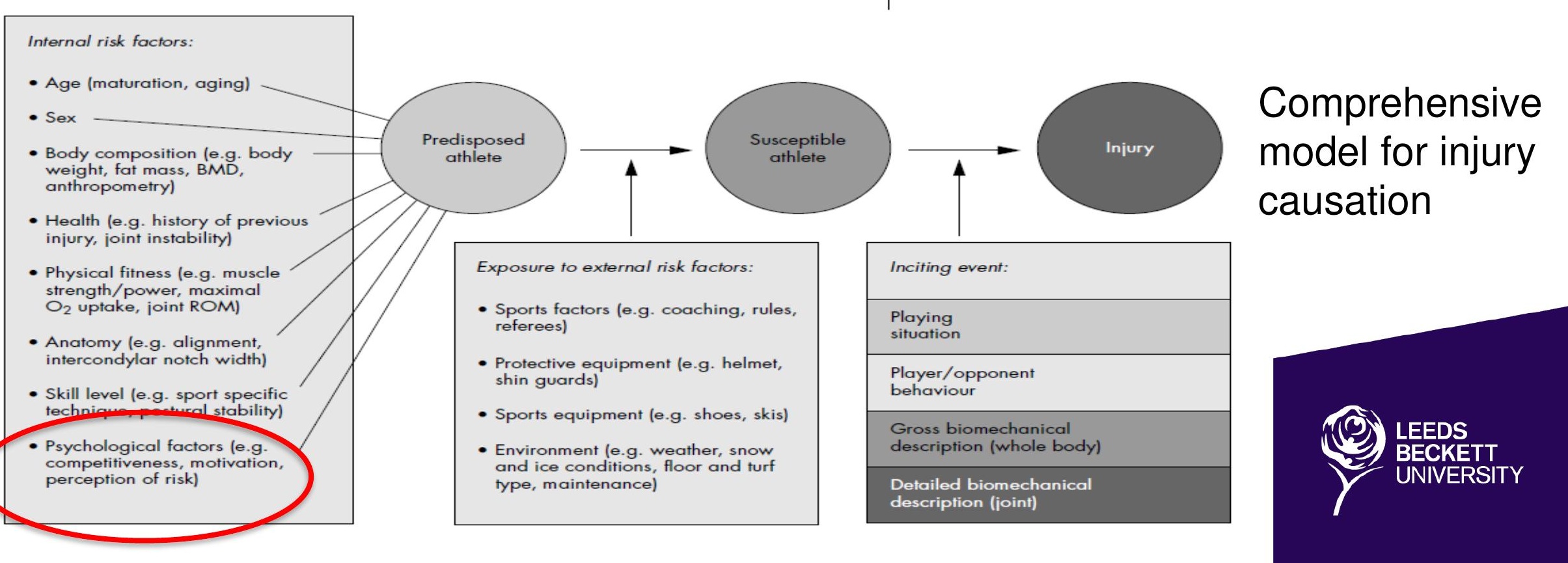




\section{Introduction}

- However...

Multifactorial causation $\neq$ multifactorial prevention?

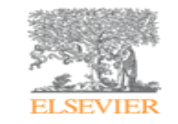

Physiotherapy

Volume 101, Issue 2, June 2015, Pages 95-102

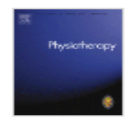

Systematic review

Musculoskeletal physiotherapists' use of psychological interventions: a systematic review of therapists' perceptions and practice

Jenny Alexanders ${ }^{\mathrm{a}} \cdot \mathbf{\sim} \cdot \mathbf{\text { , Anna Anderson }}{ }^{\mathrm{b}}$, Sarah Henderson ${ }^{\mathrm{a}}$

Physical Therapy in Sport

Volume 23, January 2017, Pages 99-104

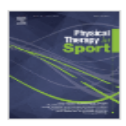

Original Research

Is there a link between previous exposure to sport injury psychology education and UK sport injury rehabilitation professionals' attitudes and behaviour towards sport psychology?

Caroline A. Heaney a . $\square$, Claire L. Rostron, Natalie C. Walkerb, Alison J.K. Green + Show more

\section{$@$ gleds13}

431

4

Crosseéc ctations

1

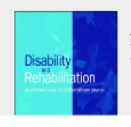

Disability and Rehabilitation

Latest Articles

Original Articles
Physiotherapists' perceptions of sport psychology intervention in professional soccer Caroline Heaney

Enter keywords, authors, DOI etc.

\section{Review Article}

0

Crosseef citionons 8 Atrmetric
Knowledge, behaviors, attitudes and beliefs of physiotherapists towards the use of psychological interventions in physiotherapy practice: a systematic review

christina Driver $\mathbf{\Xi}$. Bridie Kean, Florin Oprescu \& Geoff P. Lovell

Time constraints?

Resource constraints?

Uncertainty?

Intolerance?

Perceived benefits?

LEEDS

BECKETT UNIVERSITY 


\section{Research questions}

(1) What practical recommendations can be made for clinical practice?

(2) What is the overall methodological quality of included studies?

(3) What are the salient future research directions to advance this research area? 


\section{Method: Systematic review}

- PRISMA guidelines

- Data sources: CINAHL, MEDLINE, PsycARTICLES, PsycINFO, SPORTDiscus, Science Direct and PubMed

- Bibliographic screening

- Forward citation searching

- Hand searching of relevant journals

- Mixed-methods Appraisal Tool (Pluye et al. 2011)

- Three independent reviewers (sport psychology, sport science and sports therapy perspectives)

- Inter-researcher reliability of appraisals $=.982$ 


\section{Results: RQ1}

What practical recommendations can be drawn for clinical practice? All included studies $(\mathrm{N}=14)$ demonstrate the potential for injury risk reduction

- Cognitive/somatic relaxation $(n=6)$

- Imagery (relaxation) $(n=3)$

- Stress inoculation training $(n=5)$

- Goal setting $(n=3)$

- Attribution training $(n=1)$

- Confidence training $(n=2)$

- Self-talk $(n=1)$

- Autogenic training $(n=1)$

- Mindfulness ( $n=1)$

- Awareness training $(\mathrm{n}=1)$

$@ g l e d s 13$

- No two studies have the same intervention

- Different session/intervention lengths

- Few provide sufficient detail to replicate interventions 


\section{Results: RQ1}

What practical recommendations can be drawn for clinical practice?

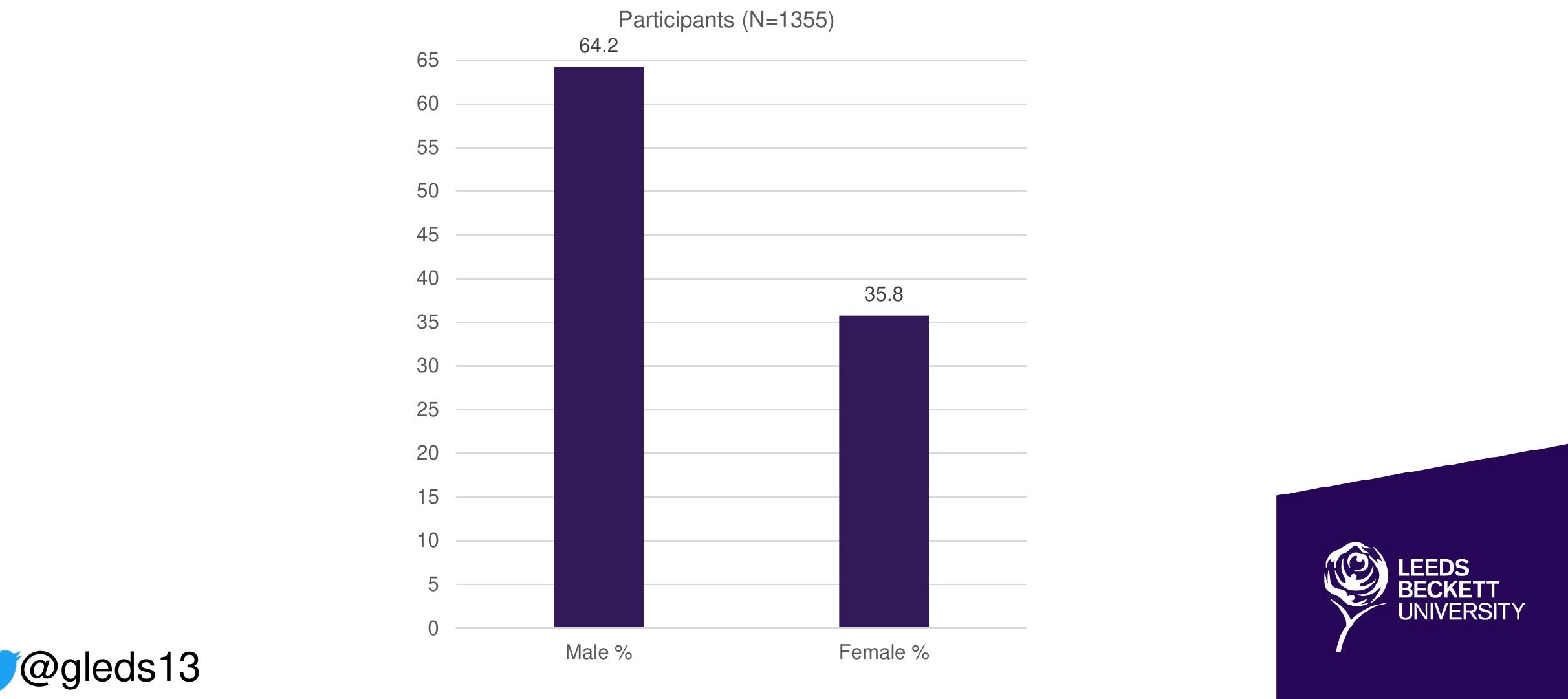




\section{Results: RQ1}

What practical recommendations can be drawn for clinical practice?

$\%$ of total participants by sport

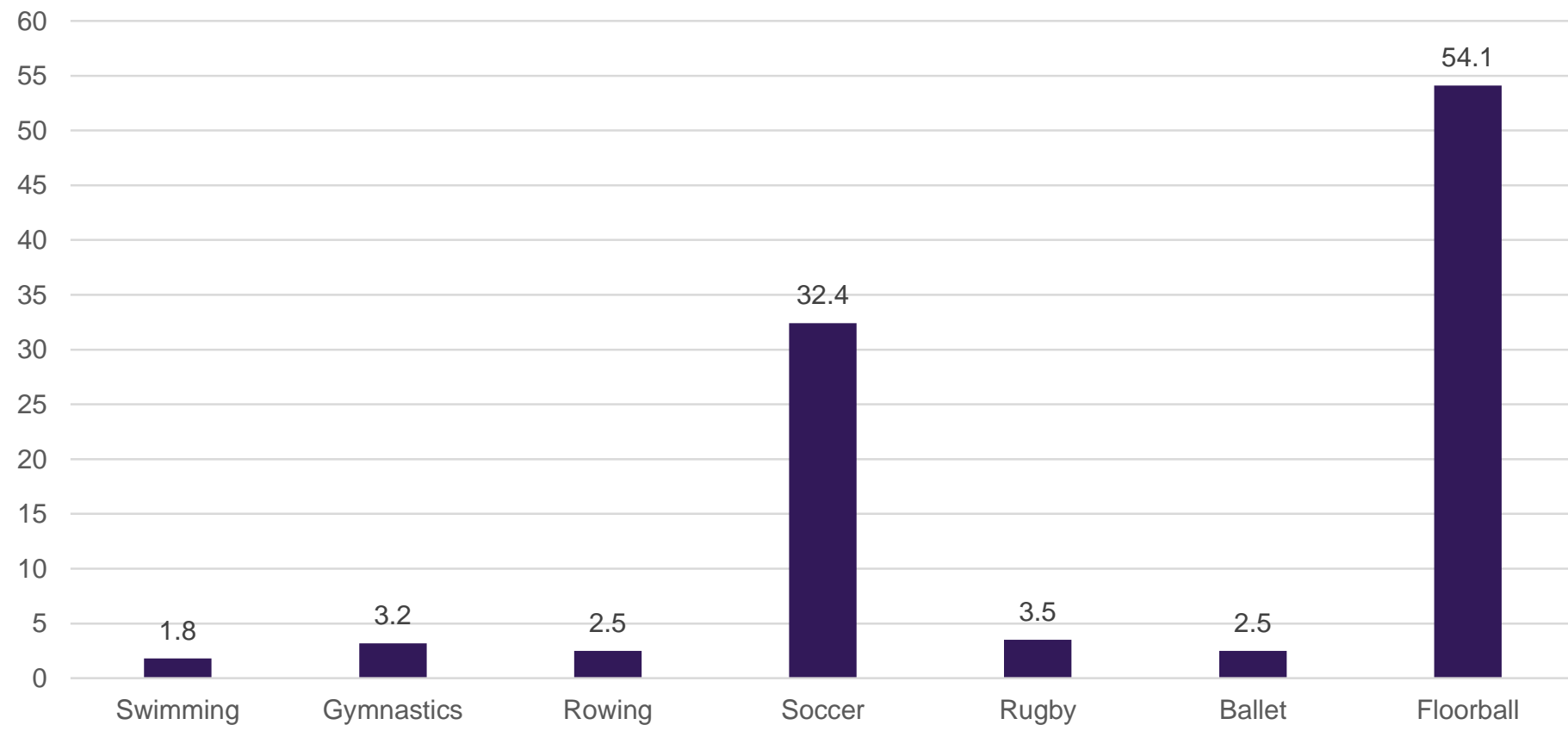




\section{Results: RQ2}

What is the overall methodological quality?

- Moderate risk of bias (51.9\%)

- Potential selection bias (e.g. selecting 'at risk' athletes for intervention groups)

- Potential reporting bias (e.g. incomplete details over randomisation)

- Concerns over sample size in $78.6 \%$ of studies 


\section{Results: RQ3}

Future research directions?

- Replication studies

- Psychological skills training aimed at eliciting neuromuscular benefits

- Psychological skills training aimed at enhancing movement quality/skill learning

- Greater representation of female athletes 


\section{Take home messages}

Where are we now?

All included psychological intervention studies demonstrate the potential to reduce injury risk

What are the existing concerns?

- Moderate risk of bias (selection and reporting)

- Lack of replication studies

- Concerns over small sample sizes in most studies

Where do we go from here?

- Future replication of stress-based studies

- Interventions which can elicit movement quality learning and/or neuromuscular changes may advance the area 


\section{Thank you for listening!}

\section{Any questions?}

\section{@gleds13}

This dissemination was supported by the Leeds Beckett University Carnegie School of Sport staff development fund 